\title{
Self in psychotherapy: An Indian perspective Jyotsna Agrawal*
}

*Associate Professor, Department of Clinical Psychology, NIMHANS. Email id: jyotsna.agl@gmail.com; Telephone: 080-26995862

\begin{abstract}
This paper discusses the two ways in which the concept of 'self' has been discussed in the Indian tradition, and its relationship to suffering and healing. There being outer and inner self, denoted as antaratman and ahamkara respectively, is a common theme across Indian darshana/ philosophy, although the exact terms and few nuances differ amongst them. Ahamkara, or the outer self seems to have overlap with concepts like ego, and self from the modern psychology. Kumar's model of Ahamkara (2005) has four main sub-components; Vaishisthya / individuality, Dwaita bhav / separation, Abhimana/ identification and Kartatva/ agency. The article describes the results from multiple studies to support such an Indian model of self, its expansion to include a componenet of 'ripe-ego', and ahamkara's association with well-being in modern world. It then goes on to discuss the implications of this Indian model of self in the psychotherapy practice along with giving a case example and future directions for further research.

Key words: Self, Psychotherapy, Indian psychology, Yoga
\end{abstract}

\section{Introduction}

Mental disorders affect almost one in seven people in India, and in less than three decades (between 1990 to 2017), their contribution to the total disease burden has nearly doubled ${ }^{[1]}$. 
Nevertheless, access to mental health services is limited. While 150 million Indians are in dire need of mental health intervention, only 30 million have access to such care, which is about one out of every five in need of these services ${ }^{[1]}$. There is a significant lack of trained mental health professionals in India, where against the actual requirement of around 54,750 such professionals, only about 7,000 are available, which is also concentrated in major cities ${ }^{[1] .}$ Although modern psychotherapy has been reasonably successful in helping people in India, and the entire spectrum and variety of psychotherapies are being practiced currently. However, given the time and laborintensive nature, its impact has been limited, especially considering India's population size. The other limitation of psychotherapy is related to the fact that established treatments may not be fully effective for everyone. For example, less than half of those treated with CBT recover from their illness. Some CBT components may not fit with some people due to personality, goals, values, resources, and lifestyles ${ }^{[2]}$. Another finding reported in the literature is that the approaches useful for treating the acute phase are not always helpful in preventing relapse or lead to full recovery ${ }^{[3]}$. Thus, newer therapeutic modalities, sensitive to and even rooted in the Indian culture, needs to be developed. This is especially true since India has a long and rich tradition of psychological ideas, many of which have already seeped into modern psychotherapy through indirect routes, benefiting many. This trend needs to be taken further in the spirit of building local psychological interventions for local problems.

\section{Culture and Mental health}

The ideas related to suffering and abnormality, its expressions and explanations, are often influenced by culture $^{[4]}$. Therefore, psychotherapeutic approaches developed in a predominantly western, individualistic context have been questioned for possible ethnocentricity and limitations 
for universal applications. When both the client and therapist share a common and culturally normative model of illness and healing, it leads to better rapport, more acceptance, and shared meaning ${ }^{[5]}$. This, in turn, contributes to those non-specific factors, which are the foundations of successful therapy. As Jacob \& Kuruvilla ${ }^{[5]}$ puts it very well, "Psychotherapies are at their weakest when they attempt to provide explanations across cultures (top-down) and are at their strongest when they are used as vehicles for engagement with patients (bottom-up)." Thus, there is a need for culturally sensitive and responsive therapies, which may lend themselves more easily to task-shifting in a public mental health approach.

\section{Psychotherapy in Indian culture}

Historically in India, something akin to psychotherapy was done by gurus and healers ${ }^{[6]}$. While sick went to healers (e.g., Ayurveda, Siddha practitioners, etc.), gurus predominately focused on psycho-spiritual growth and self-realization of people. The commonality was that both required respect and belief ${ }^{[6]}$. In the Indian context, the gurus may be considered leaders in a preventive mental health approach due to their focus on yogic pathways to personal and spiritual development. Further, these interventions were often rooted in India's nine major ancient philosophical-spiritual traditions, known as 'Darshana' (Sanskrit term translated as 'seeing' or

perspectives), which includes Yoga, Vedanta, Buddhism, Jainism, etc. Balodhi and Chowdhari ${ }^{[7]}$ have summarised a variety of interventions from Atharva-Veda. It is also noted that the variety of psychological interventions in the Indian tradition are spread on a continuum of directive versus non-directive approaches (Diagram 1).

Here it is relevant to emphasize that the term yoga has two meanings, one as a specific school of Indian philosophy, which was coded by Patanjali in his Yoga Sutra. Secondly, this 
term is mainly used to denote all those techniques which people practice to find release from their existential sufferings and achieve unity consciousness ${ }^{[8]}$. In this later usage, yoga may be considered a collection of inner technologies or 'technologies of the self'(Foucault $\left.{ }^{[9]}\right)$. Some yoga components have been regularly utilized in mental health services, such as body-based 'yogasana,' breathing practices, meditations. Furthermore, there has been much empirical literature on yoga's benefits in various mental health problems to support this. The psychological components of this tradition related to attitudes, cognitions, emotions, actions, etc., are yet to be fully utilized in such interventions. One of the psychological aspects is that of ideas related to 'self,' which will be discussed in more detail below.

\section{Self in the Indian tradition}

In a thematic review of three yogic texts (Patanjali Yoga Sutra, Bhagawad Gita, and Yoga Vashistha), considered to be core ${ }^{[10]}$, it was found that Self is seen as both outer and deeper ${ }^{[11]}$. The deeper Self, often denoted with capital'S', has been described as the pure consciousness and unaffected witness within, and often termed as Antaratman, Chaitya-purusha, etc. This Self is considered as the imperishable spark of Divinity in a being and remains in oneness and identification with the universal Self, which also connects it with all others. The Outer self (written with small 's') is ahamkara, the body-based identity, and encompasses various concepts of modern psychology, such as self, ego, and identity. It has been described using the metaphor of a protecting cover (e.g., pot, house, etc.) for inner space. This outer self is mind-based and can color one's perceptions with self-relevant contents (beliefs, images, feelings ${ }^{[12]}$. It is limited due to a sense of separation, ownerships, and attachments, pride about 
one's uniqueness, and feeling in control of one's actions ${ }^{[13]}$. Based on Vedantic conceptions, Kumar et al. ${ }^{[13]}$ named these four components as:

(a) Vaishisthya / individuality, related to a sense of being unique

(b) Dwaita bhav / separation, with a feeling of being different from others

(c) Abhimana/ identification, related to one's associations, belongingness, and possession

(d) Kartatva/ agency, about one's sense of doer-ship control and personal efficacy

An example of this would be a person who is doing well professionally, leading to a strong sense of doer-ship (kartatva) along with an inflated self-image (vaishisthya). He may be attached to his membership of various associations (abhimana) and may also feel different from other professions (dvaita bhava). These components may interact with each other to enhance a person's outer self, making him live within his small subjective bubble until there is a problem in his professional life.

\section{Outer self and suffering}

The Indian tradition considers the cause of suffering is due to living at the outer self level, focused on the external world or getting trapped in its complexities, and being ignorant of one's deeper Self. On similar lines, Pandey ${ }^{[14]}$ mentions, "The source of our psychological maladies is an inability to live deeper." He then adds, "with this sense of surface' me' also comes the sense of 'not me.' No real unity or harmony with the world around is possible with the ego, only some accommodation, tolerance, and adjustment." ${ }^{14]}$

Yogi-mystic Sri Aurobindo ${ }^{[15]}$ considered this outer self as both a helper and a bar. Initially, such ego-sense helps in individuating from the common mass of humanity. However, as 
one progresses further on spirituality, he goes beyond this outer self to find one's inner Self, which is also the center of true individuality. Ordinarily, most people may feel just fine while living at the outer self level, especially when their lives are going well with its combination of joys and sufferings. Until inner or outer circumstances force them to change and grow, they do not voluntarily take this path.

\section{Healing and growth}

To overcome suffering and to experience psychological growth and healing, the Indian tradition proposes connecting with the ultimate Divine reality by minimizing and finally transcending the outer self. This happens in parallel with recognizing and getting closer to the unaffected witness, the deeper Self inside. In the process, one can know the connection betweenself-other-universal Self, where there is a sense of oneness and interconnectedness (between the individual and/or the Immanent, Cosmic, Transcendental aspects of Divine). Initially, this may only be an intellectual understanding, but over time, with practice, this can become a lived reality. This is especially true since the mind is considered both, like an enemy or a friend (Bhagawad Gita). Here the six enemies of lust or desire (kama), anger (krodha), greed (lobha), attachment (moha), arrogance or pride (mada), and jealousy (matsarya), when given free play, can strengthen the outer self.

On the other hand, the mind can also help when it consciously chooses to grow, (considered as ripe ego by Sri Ramakrishna Paramhansa ${ }^{[16]}$ ) and finally dissolves itself and the

outer self in the universal consciousness. Mystic Sri Ramakrishna Paramhansa ${ }^{[17]}$, had used the metaphor of 'salt doll' which dissolves in the ocean water to describe such dissolution of the 
outer self. This is probably from where the popular idea of 'oceanic feeling,' popularly used in transpersonal psychology, originates.

\section{Pathways to healing}

Multiple yogic pathways are given in the Indian tradition, the trimarga of jnana, bhakti, and karma, being the three main broad paths. Each path can dissipate the outer self, bring forth the inner Self, and result in wisdom and clarity. This requires some separation from our daily preoccupations with the external world and ahamkara and involves de-identifying from the outer self, self-observation, and analysis. It is an experiential journey in a broad time-space framework

(across births), and it is believed that no effort is $\operatorname{lost}^{[18]}$. Simultaneously, one needs to discover a harmonious way of co-existence till one realizes a deep oneness and interconnectedness with all creation, as the same Divinity is present in all. This can be achieved by following specific socialethical codes (such as satya, ahimsa, asteya, brahmacharya, aparigraha). By cultivating positive interpersonal attitudes (bhavana or brahmavihara of maitri/ friendliness, mudita/ appreciation, karuna/ compassion, and upeksha/equanimity), while also working towards social welfare (lokasamgraha).

\section{Empirical research}

Multiple studies, using a mixed-method approach, have been conducted, to understand the concept of self in modern times. The model of ahamkara based on Vedantic literature was validated by the emergent themes in one of our mixed-method studies ${ }^{[19]}$. It reported that the research participants had self-construal themes on the lines of Individuality, Separation, Agency, and the Belongingness aspect of the Identification, as per the Ahamkara model ${ }^{[19]}{ }^{[13]}$. Further, a 
new category of Spiritual/Transpersonal self-construal also emerged, which was in line with what Sri Ramakrishna Paramhansa spoke as 'ripe ego. ${ }^{\text {[16] }[19]}$ Further, both qualitative and quantitative results in multiple studies have found that ahamkara is higher in young adults, in men and single people, and it decreases with age, although different component seems to have different trajectories. ${ }^{[19][20] ~[21][22] ~}$

Overall, ahamkara is associated with lower well-being, however it differs with different subcomponents. Sense of identification to specific groups and things (belongingness) was associated with lower well-being, Individuality (higher in men) had mixed results related to positive and negative affect, and agency (higher in men and middle age) was positively associated with well-being. ${ }^{[19][20][21][22]}$ In one study, ${ }^{[22]}$ ahamkara was positively correlated with wisdom. Such mixed results support the comment by Sri Aurobindo ${ }^{[22]}$ that the ego can be both, a helper and a hurdle. The yogic traditions acknowledge that a healthy ego is required initially, since a moderate amount of ahamkara helps in managing life demands and allows a person to function in the transactional world. The natural trajectory seems to be that it diminishes with age.

When a person consciously practices the yogic pathways resulting in psycho-spiritual growth, the ahamkara-driven self-focus decreases. This stage is similar to the idea of 'quiet ego' in mainstream psychology. One study ${ }^{[21]}$ reported such quiet ego was negatively correlated with individuality aspect of ahamkara and positively correlated with character strengths. This brings up the next question regarding, how to quieten the ego? The community adults responded that for a healthier state of self, a person needs to decrease preoccupation with oneself, decrease their sense of separation, not diminish others, give importance to others and focus on developing positive qualities and spirituality within. ${ }^{[21]}$ 


\section{Clinical implications}

The larger goal of psychotherapy can go beyond just removing symptoms and getting closer to one's true Self, the healer within as per the Indian tradition. ${ }^{[14]}$ However, most psychotherapists may not feel competent enough to work on such a broad and long term goal. Thus balancing various outer self/ ahamkara components in the psychotherapy practice may be attempted. The need for balance in the outer self arises since a heightened sense of separation may lead to conflicts with others. Excessive individuality may make a person come across as selfish and self-absorbed. In contrast, strong attachments can make one prone to intense grief, and a high sense of agency may make it challenging to absorb failures. Thus, working on all these will improve the mental health of a client in psychotherapy. Developing a spiritual self or 'ripe ego' may likely help in such situations, even if it is mostly cognitive and not experiential, initially. Future research can also study the operations of these sub-components in different mental illnesses.

Additionally, strengthening of the outer self may be required in some cases, such as in psychosis. In one such case, a young woman suffering from delusional memory developed insight through meta-cognitive therapy, which made her vulnerable to shame and distress about her past behavior and soon led to another relapse. An innovative therapeutic approach was then undertaken, focusing on strengthening the outer self's different components and connecting it with time and space. In the Indian tradition, the sense of time, space, and context (desh, kaal, paristhiti) are secondary components supporting one's outer self in a transactional world. Time and space have also been linked to a sense of boundaries. ${ }^{[23]}$ Given the client's delusional memories, it was considered essential to work with time and space in this case. The patient 
showed improvement after a few sessions and was discharged. More research is needed in this area to strengthen this hypothesis.

\section{Summary}

In summary, based on insights from the Indian tradition and the texts, an interconnected model of self, which has outer, deeper and interconnected aspects, may be considered for psychotherapy. This may be useful since even in modern India, those giving less importance to the outer self seems to have higher well-being. Thus, psychotherapy having an individualistic assumption may be prone to cultural mismatch in India and may even lower well-being over time. Psychotherapy, which gives importance to the deeper self, relational harmony, and balance, may be a better approach in the Indian context.

\section{References:}

1. Sagar R, et al.,. The burden of mental disorders across the states of India: the Global Burden of Disease Study 1990-2017. The Lancet Psychiatry. 2019;7:148-61.

2. Vittengl JR, Clark LA, Dunn TW, et al. Reducing relapse and recurrence in unipolar depression, a comparative meta-analysis of cognitive-behavioral therapy's effects. Journal of Consulting Clinical Psychology. 2007; 75(3):475-488.

3. 2. Driessen E, Hollon, SD. Cognitive behavioral therapy for mood disorders: efficacy, moderators, and mediators. Psychiatric Clinics of North America. 2010; 33(3); 537-555.

4. U.S. Department of Health and Human Services. Mental Health: Culture, Race, and Ethnicity-A Supplement to Mental Health: A Report of the Surgeon General. Rockville, MD: U.S. Department of Health and Human Services, Substance Abuse and Mental Health Services Administration, Center for Mental Health Services. 2001. 
5. Jacob KS, Kuruvilla A. Psychotherapy across cultures: the form-content dichotomy. Clinical psychology \& psychotherapy. 2010. https://doi.org/10.1002/cpp.736

6. Neki JS. Psychotherapy in India: past, present, and future. American Journal of Psychotherapy. 1975; 29(1):92-100. DOI: 10.1176/appi.psychotherapy.1975.29.1.92

7. Balodhi JP, Chowdhari JR. Psychiatric concepts in Atharva Veda: A review. Indian Journal of Psychiatry. 1986; 28: 63-68.

8. Larson GG, Bhattacharya RS. Encyclopedia of Indian Philosophies (Vol. 12): Yoga: India's Philosophy of Meditation. Motilal Banarsidass; 2nd edition. 2016.

9. Foucault, M. https://en.wikipedia.org/wiki/Michel_Foucault

10. Salagame KK. Indian indigenous concepts and perspectives: developments and future possibilities. In G. Misra (Ed.), Psychology in India: Theoretical and methodological developments- ICSSR survey of advances in research, Vol. IV. New Delhi: Pearson. 2011, pp. 93-172.

11. Agrawal J. Yogic tradition and well-being. Unpublished observations. 2020.

12. Mills KL. Experiential self-referential processing \& autobiographical memory retrieval: A preliminary look. PSU McNair Scholars Online Journal. 2011; 5, 198-221.

DOI:10.15760/mcnair.2011.190

13. Salagame KK, Raj A, Murthy PK, Parimala, Rekha, Gaur S. Concept of Ahamkara: Theoretical and Empirical Analysis. In K. R. Rao and S. B. Marwaha (Eds.) Towards a spiritual psychology: Essays in Indian Psychology. New Delhi: Samvad India Foundation. 2005, pp. 97122

14. Pandey, A. Psychotherapy and Indian Thought - III. 2010. Available from https://nextfuture.aurosociety.org/psychotherapy-and-indian-thought-3 
15. Sri Aurobindo. 1956. Thoughts and glimpses. SABCL; 16, pp. 377. Available from http://library.sriaurobindoashram.org/mother/cwm08/chapter/49/

16. Swami Shraddhananda. The ego and the self. Available from https://vedanta.org/2003/monthly-readings/the-ego-and-the-self/

17. Sri Ramakrishna Paramhansa. A salt doll went to fathom the ocean. Available from https://www.ramakrishnavivekananda.info/parables/1_files/1-122.html

18. Dalal AK, Mishra G. The core and context of indian psychology. Psychology and Developing Societies. 2010; 22 (1), 121-155. https://doi.org/10.1177/097133360902200105

19. Gupta K, Agrawal J. Ahamkara: A study on the Indian model of self and identity. Unpublished observations. 2019.

20. Gupta K, Agrawal J. Anasakti and ahamkara in relation to mental health. Unpublished observations. 2020.

21. Rai V, Agrawal J. Association of quiet ego, ahamkara and anasakti with character strengths and psychological well-being among emerging adults and middle-aged adults. Unpublished observations. 2019.

22. Smrithi, Kapanee AM, Agrawal J. Association of Kleshas and Brahmaviharas with Psychological Distress and Wisdom among College Going Youth. Unpublished observations. 2017.

23. Ataria Y, Dor-Ziderman Y, Berkovich-Ohana A. How does it feel to lack a sense of boundaries? A case study of a long-term mindfulness meditator. Consciousness and Cognition. $2015 ; 37,133-147$. 
Diagram 1: Directive vs. non-directive interventions in the Indian tradition

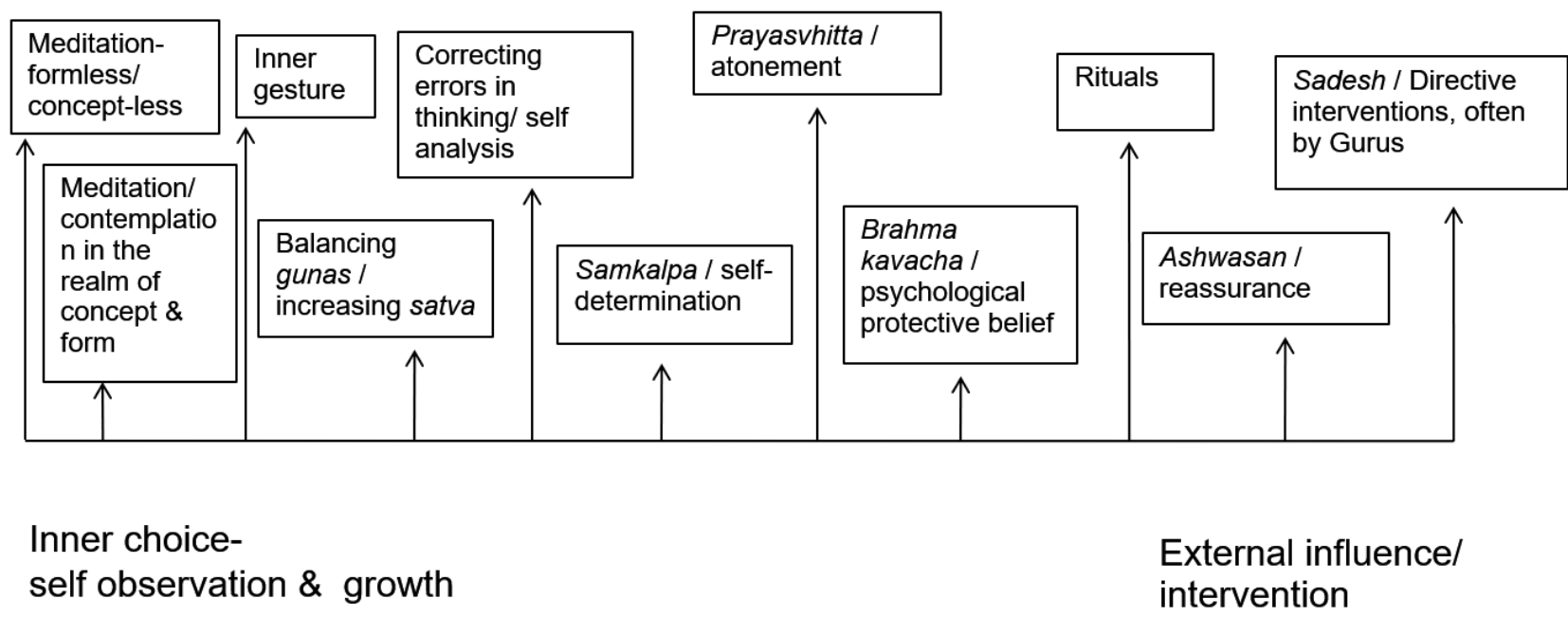

Continuum of interventions based on Indian Psychology 